\title{
Tendencies in Multi-Agent Systems: A Systematic Literature Review
}

\author{
Mariana Falco \\ Universidad Austral, Facultad de Ingeniería, LIDTUA/CONICET \\ Pilar, Buenos Aires, Argentina, 1623 \\ mfalco@austral.edu.ar \\ and \\ Gabriela Robiolo \\ Universidad Austral, Facultad de Ingeniería, LIDTUA \\ Pilar, Buenos Aires, Argentina, 1623 \\ grobiolo@austral.edu.ar
}

\begin{abstract}
The application of Artificial Intelligence mechanisms allows the development of systems capable to solve very complex engineering problems. Multi-agent systems (MAS) are one paradigm that allows an alternative way to design distributed control systems. While research in this area grew exponentially before 2009, there is a need to understand the status quo of the field from 2009 to June 2017. An extension of the results of a SLR related to Multi-Agent Systems, its applications and research gaps, following Kitchenham and Wholin guidelines are presented in this paper. From the analysis of 279 papers (out of 3522 candidates), our findings suggest that: a) there were 20 gaps related to agent-oriented methodologies; coordination, cooperation and negotiation; modelling, developing, testing and debugging; b) 24 gaps related to specific domains (recycling, dynamic evacuation, hazard management, health-care, industry, logistics and manufacturing, machine learning, ambient assisted living); and 14 gaps related to specific areas within MAS (A-Teams, dynamic MAS and mobile agents, ABMS, evolutionary MAS, and self-organizing MAS). These gaps specify lines of research where the MAS community must work to achieve the unification of the agent-oriented paradigm; as well as strengthen ties with the industry.
\end{abstract}

Keywords: Systematic Literature Review, Multi-Agent Systems, MAS Research Gaps, AOSE components.

\section{Introduction}

Over two decades ago, agents and Multi-Agent Systems (MAS) became a novel way of conducting the analysis, design, model and implementation of complex software systems [1]. Agents can be defined as computer systems, autonomous, and they can exhibit a flexible behaviour (to be reactive, proactive and to achieve social ability) [2]. This definition is extended by the Belief-Desire-Intention (BDI) notion [3]. A MAS contains an environment, objects and agents, relations between all the entities, a set of operations that can be performed, and the changes of the environment in time and due to these actions [4].

As a result of the growing development of relevant technologies, and the research done within the past years, agent-based systems become hailed as a new paradigm or an emerging paradigm, which is capable of many societychanging practices [5]. Other authors [3] propel the need of justifying the paradigm shift from paradigms like the object-oriented (OOP) to the agent-oriented (AOP). The engineering of MAS is a complex activity due to be implemented as a distributed system consisting of multiple agents that work together in order to solve common problems. As such, different studies have been carried out to provide a comprehensive background for specifying, designing, and implementing MAS [6-11]. Mostly, the conducted studies are rather specific than general; and so we have considered necessary to understand as well as summarize the progresses and research gaps within MAS field. The main goal was to conduct a SLR around six research questions, concerning the representative authors, the 
description of the applications and their domains, AOSE components like methodologies and frameworks, and research gaps, from 2009 to June 2017 in the point of view of practitioners and researchers, in the industrial and academic context. We followed the guideline provided by Kitchenham [12], complemented with the snowballing approach [13], which begin with a set of relevant papers which will be later analyse by their citations and references in order to add papers to the original set. From the four original papers, we obtained 3522 articles through the application of the snowballing approach, and after the inclusion/exclusion criteria, only 279 articles remained.

The two main characteristics of the articles were as follows: a) dispersion based on amount of publication venues, domains; methodologies, frameworks and architectures; and b) low quantity of applications on real cases of real settings. The present study is an extension of another work by the authors [14]; and in this case, the focus was placed on the research gaps, reviewing the selected articles again and incorporating a greater number of gaps, both general and specific, into the tables; which is a huge contribution to the MAS community, achieving 20 gaps related to agent-oriented methodologies; coordination, cooperation and negotiation; modelling, developing, testing and debugging; b) 24 gaps related to specific domains (recycling, dynamic evacuation, hazard management, health-care, industry, logistics and manufacturing, machine learning, ambient assisted living); and 14 gaps related to specific areas within MAS (A-Teams, dynamic MAS and mobile agents, ABMS, evolutionary MAS, and self-organizing MAS). As a result, we strongly recommend to the MAS community that they increase their work together to unify the field, extending the bridge with other disciplines and the industry. And this would also lead to increase the amount of applications applied on real cases.

This article is organized as follows: Section 2 provides a background with previous SRLs, while Section 3 describes the systematic literature review method. Section 4 describes the results and findings. Section 5 explains the threats to validity, while Section 6 provides a brief discussion, and finally, Section 7 presents the conclusions and future work.

\section{Related Work}

Considering that agents are able to shape the society in which they interact, through argumentation techniques is possible to model those interactions. Carrera and Iglesias studied argumentation techniques from 1998 to 2014, and, they have concluded that "(...) the argumentation technology is actually in a phase of internal exploration (...)" [15]. Later on, two of the most differential aspects of agents are their autonomy, and that they are embedded in a highly dynamic environment. Some researchers introduced the Adjustable Autonomy, which allows a system not only to operate in different autonomic conditions, but also to transfer control between the system's operators. Mostafa and others described that there is no specific model or algorithm that yields viable adjustable autonomy [16]. Remembering that a reactive system must respond by taking actions to several changes that occurs in its environment; the Teleo-Reactive paradigm allows developing reactive systems like robotic vehicles. In this context, Morales and others pointed out that it is a highly promising subject of study, and that require interdisciplinary collaboration with Software Engineering (SE) [17].

Likewise, Agent Oriented Software Engineering (AOSE) allows to apply the principles from SE and AI to the life cycle of a software system [8]. Blanes and others identified the Requirements Engineering (RE) techniques that has been applied while developing MAS, and they found out that $79 \%$ of the papers used methods or techniques from existing paradigms; and that $69 \%$ of these methods and techniques were built on the goal-oriented paradigm [17]. Similarly, Juziuk and others found out only 206 Design Patterns (DP) since 1998, from that they have concluded that there is no standard template to help with the process of DP documentation for MAS, the associations between patterns are poorly described and the classification of patterns are tied to specific DP lists [19].

There have been domain-specific studies within MAS field, such as health-care and marketing. In the first case, Isern and Moreno showed the viability of applying and implementing agents within the health-care domain, and from which it can be concluded that agents applied in health-care is a growing field. In the second case, Negahban and Yilmaz provided an analysis in marketing, where they pointed out that several Agent-Based Modelling and Simulation (ABMS) application within conventional markets and new product diffusion have been described in a high number of studies; demonstrating the capability of ABMS to capture and model emergent phenomenon [20].

As can be seen in the previous descriptions, systematic studies address research questions on a specific topic such as argumentation techniques or design patterns; from which it is possible to say that the decreasing trend of publications does not affect specific domains or modelling and simulations. However, we have not found studies that share the same scope as the present work, which approaches the identification from 2009 to June 2017 of the progresses, AOSE components, MAS applications, domains, representative authors, and gaps. The following sections will describe the review method and the answers to the research questions. 


\section{Review Method}

The present SLR followed the guidelines provided by Kitchenham [12], and complemented through the search approach known as snowballing defined by Wohlin [13].

\subsection{Research Questions}

According to the goal presented, we formulate the following set of Research Questions (RQs), where RQ1 concerns to the general objective, RQ2 and RQ3 allow identifying and characterizing authors and communities, while RQ4 to RQ6 address topics related to applications, and finally RQ7 allows the identification of SMA gaps. The list of RQs is the following: Research Question $1(R Q 1)$ : What are the articles that represent an up-to-date SMA state-of-art?; $R Q 2:$ Who are the most representative authors?; $R Q 3:$ What are the main publication venues?; $R Q 4$ : What are the most applied methodologies, frameworks, and programming languages?; $R Q 5$ : What are the most selected application domains?; and RQ6: What are the research gaps?

To address RQ1, we performed the review method described earlier, in order to find those relevant papers that will lead us to obtain a proper collection of the published projects or researches from 2009 to June 2017 within the MAS field. Also, 2009 was considered as the starting point due to the publishing of a book [2] (with a growing number of citations - 11047 to January 2, 2019, and a verifiable background of the authors - see [1], [21]) which provided an introduction and compilation of all the main theoretical and practical topics from intelligent agents and MAS.

\subsection{Inclusion and Exclusion Criteria}

Articles published between 2009 and June 2017 were included if they fulfilled the following topics: (a) the title and/or the keywords (if exists) should contain "multi-agent systems", "multiagent systems", "intelligent agents", "agents", "MAS" or similar phrases, (b) studies which were directed related to MAS techniques, approaches, challenges, implementations, gaps or limitations; (c) studies that were directed related to AOSE components; (d) studies related to MAS implementations and case studies applied to different domains; (e) studies whose type were surveys, systematic reviews, review, case studies, formalization, applications. Papers on the following topics were excluded: (a) studies on a non-English language; (b) duplicate or updated studies (we selected the most recent one); (c) journals with low impact factors; (d) books; (e) extended abstracts; (f) technical reports; (g) doctoral dissertations; and (h) thesis.

\subsection{Search Strategy}

In order to obtain a proper set of relevant papers for this review, we conducted a manual search, an automated search and the snowballing approach.

\subsubsection{Manual Search}

One of the authors performed a scan of publication venues and journals, to attain a panorama of communities and publishers in subjects related to multi-agent systems. From this search, systematic reviews and books were obtained that allowed us to put together the RQs, as well as the inclusion and exclusion criteria. Also, the Cohen's Kappa statistic [22] was used to perform an evaluation of the reliability of the inclusion and exclusion decisions between the raters (the authors), which gave a value of 0.75 , showing the level of agreement.

\subsubsection{Automated Search}

After the manual search, we queried Google Scholar based on [10], using \{systematic review; multi agent systems\}, and we judged the relevance of the results to the RQs based on title, and publication year. In due consideration that the content of the databases and index vary over time, the actual search was conducted on June 2017. As a result of this step, one of the authors identified 9 candidates for inclusion (reviewed by the other one). Even though Giustini and Boulos [23] pointed out that "Google Scholar does not meet the required search standards for conducting a $S L R$ ", our main target was the next step (snowballing) in order to discover patterns and update the knowledge on the field. The 9 articles were analysed regarding the abstract, references and citations; from which 4 articles were selected to form the start set, denoted as S1 to S4.

\subsubsection{Snowballing}

Following [13], we put together the initial point of analysis: S1 had 74 references and 4 citations; S2 32, and 3; S3 161, and 3178; and, finally S4 34, and 36. As is it possible to observe, the search produced a large number of potentially relevant papers (3522 to be exact). These papers were assessed for inclusion or exclusion against the 
specified criteria previously defined. Only one iteration has been performed. Articles S1, S2 and S4 were tested by implementing the iterations with the backward and forward snowballing procedures. S3 was only studied by forward snowballing due to its publication year. From S1, S2, and S4; 140 candidates were evaluated, and 16 new papers were included, denoted S5 to S21. Later, forward snowballing was applied to the full start set, leaving 3221 citations to evaluate, from which 259 were included, denoted as S22 to S279.

\subsubsection{Data Extraction and Synthesis Methodology}

We created a form to consolidate the data extraction, in which the following items were completed for each article by the two reviewers: (a) author (full name, affiliation and institutions/universities, country, amount of papers), (b) amount of citations, (c) publication year, (d) source (journal, conference, congress, workshop, symposium), (e) publisher, (f) developed applications: domain, availability, license, framework, programming language, architecture, platform, methodologies, (g) new programming language architecture, platform or methodology, (h) type of study, (i) research gaps, and (j) scope. Also, another spreadsheet was used to perform the mathematical analysis over the values and amounts. To obtain the 'most representative' authors, the articles were filtered, on the one hand for the amount of citations for each one of them, and on the other hand, for the amount of papers within the full set of papers (279). From that and in first place, we filtered the top 20 ordered from higher to lower number of citations from which we obtained 64 different authors. Secondly, by choosing each of the authors of the top 20, we correlate each of the articles in which he/she appeared as author (1 to 7 papers) with the number of citations for each of those articles; in order to obtain the sum of all the citations for the total articles of each author.

\section{Results and Findings}

\subsection{Overview of Papers}

Our findings suggest that between 2009 and 2017 there has been a few stellar years, as illustrated in Fig. 1. The year with most publications is 2009 with 65 articles, followed by the other years in ascending order, with a minor change in the list where 2015 obtain three more papers than 2014. Unfortunately, the general trend line for agent-based oriented papers is decreasing, also exposed by Balke and others [24] who examined the main track proceedings of AAMAS from 2006 to 2011 (the amount of application papers decreased from $10 \%$ to $6 \%$ ). Nevertheless, finding 4.1 shed light onto the trend (see for example [10] in healthcare).

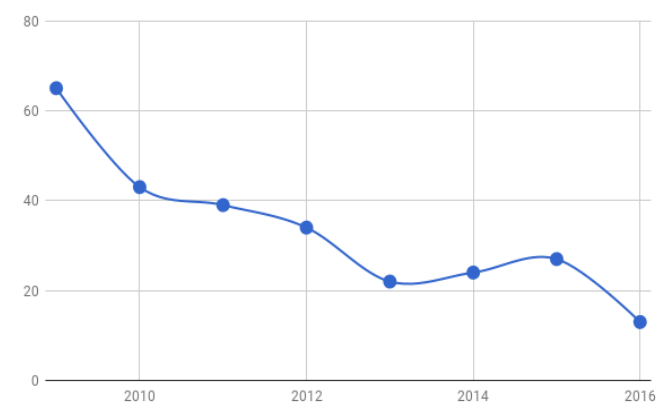

Figure 1: Trend line of analyzed articles per year

Finding 4.1. There is a general decreasing trend of publications, but an increasing trend within specific fields.

Regarding the papers, $69.8 \%$ of the selected articles were applications while the remaining $30.2 \%$ were researchers studying the MAS paradigm and performing SLRs, surveys, theoretical formalizations, among others. Within the applications, only $22.56 \%$ of the articles portrayed a real case study, and the other $77.44 \%$ were controlled case studies through simulations, evaluations, comparisons, test bed, and empirical evaluations through practical metrics; leading to obtain experimental results.

Finding 4.2. Approximately $82 \%$ of the studies were conducted within the academia, $16 \%$ in cooperation with industries and businesses, $6 \%$ without the academia.

Considering the full amount of articles, $82 \%$ of authors belonged to departments, faculties, institutes, and/or laboratories of approximately 250 universities globally distributed. While the remaining $16 \%(6 \%$ developed and owned without the academia) is represented by industry leaders such as Ansaldo Segnalamento Ferroviario; companies such as Portugal Telecom Inovação e Sistemas; councils such as the National Council for Scientific and Technical Research (CONICET); research centres such as IBM T.J. Watson; and state services such as Meteorological Service of Cyprus; just to name a few examples. 
These values showed an update on the values presented in [9], and based on an analysis of 152 applications. From $28 \%$ of applications within academia and a $41 \%$ built in industry-academic cooperation, our findings (based on 194 applications) suggest an increase of $54 \%$ on the first players, and a decrease of $25 \%$ for the second players. Nevertheless, only 10 applications provided a link to the code of the implementation, or at least to some libraries to download. Then, $70 \%$ of the application papers described the amount of agents, which varied from two to fourteen agents.

\subsection{RQ3: What are the main publication venues?}

Regarding to the 102 journals obtained, approximately $71 \%$ of the articles correspond to 48 different journals with one article each, which it is related to the affirmation that broader communities of researchers in MAS are publishing not in a few journals (and conferences, workshops), but in several and every time within more and more specific domains. The Top 3 of Journals are Expert Systems with Applications, Information Sciences, and Autonomous Agents and Multi-Agent Systems; where the Top 2 of Conferences are International Conference on Autonomous Agents and Multiagent Systems, and KES Conference on Agent and Multi-Agent Systems Technologies and Applications. Likewise, from 95 conferences, approximately $87 \%$ of them have only one article, again evidencing the dispersion. Also, the number of congresses (4) and symposiums (11) is low while the journals (102) and conferences (95) take the largest number of representatives. Each of them provide a means of discussion or practical work on a particular topic, allowing speakers and assistants to share their knowledge, experience, suggestions; due to the fact that as scientific and technological advances, it presupposes a higher level of specificity of topics and domains to achieve a feasible solution. In addition, interdisciplinary work is fundamental to that goal, because it also opens doors to unknown but rich territories.

Finding 4.3. The articles were mostly published in journals (44.7\%) and conferences $(42.7 \%)$.

\subsection{RQ4: What are the most applied methodologies, frameworks and programming languages?}

Several papers enumerated distinct and well-known methodologies such as Gaia, MaSE, O-MaSE, INGENIAS, AGR, Tropos, Prometheus, among others; but only $20 \%$ of the analysed studies explained the chosen methodology that was part of the implementation. The most used methodologies were Gaia and Prometheus. From 2009 to June 2017, new domain-specific methodologies have been formalized like mechatronic and environmental systems, traffic lights, supply chain, among others. We have found that some researchers have extended a well-known methodology based on some necessities or on problems identification within the formal definitions. On the one hand, Prometheus was extended to improve the requirements understandability, and maintainability through the automatic generation of activity diagrams [25]. On the other hand, Gaia is extended in [26] to make it more practically applicable as model-driven engineering, and extended in [27] with the security model.

Finding 4.4. There are new domain-specific methodologies, and Prometheus and Gaia have been extended.

The existence of several methodologies have stimulated the creation of multi-agent programming languages (PLs) and frameworks [28]. 44 of 195 applications provided data about the PLs used in the development. Java (not agent-oriented) has been the most popular PL used in 18 applications. C, C++, C \#, and Jason followed it. Within the agent-oriented PLs, there are individualities such as the Jazzyk language in gaming, Jadex used in bankruptcy contagion effects, and tuProlog in railway signalling.

Finding 4.5. Java was by far the most used PL in 18 applications (of 195).

With respect to frameworks, 64 of 195 applications provided information about the framework used. In our case, JADE was the most used framework out of 36 applications. Even though the sample is small (56\% out of 64 applications), our findings are consistent with what some authors have put forward about the most used frameworks [9] meaning that JADE, and JACK, remain as the most used agent development frameworks.

Finding 4.6. A) JADE was by far the most used framework in 36 applications (of 195), mainly in traffic, transport, logistics, and manufacturing; B) There are new domain-specific frameworks, as well as new frameworks for MAS themes and topics; C) Based on A), a detailed analysis of JADE is required.

Following C) within Finding 4.6, it is possible to say that even though some companies have been interested in the development of MAS, point A) is indeed an academic point of view; due to the fact that the authors have performed an analysis of existing documentation, and they did code tests and reviews. From those, some issues have been found: JADE has a deprecated Android version, it also shows deficiencies within the SOAP protocol. Likewise, there is a lack of future maintainability of any application developed with JADE, and even though it is open source it does not have a public repository on Git, and finally, it is a verbose framework [29]. 


\subsection{RQ5: What are the most selected application domains?}

The distribution of 195 applications were given by 16 different domains, which are the following: information science (D1), transport / traffic / ports (D2), health-care / medicine / biology (D3), networks (D4), logistics / manufacturing (D5), mechatronics (D6), business (D7), economy / finance (D8), earth related (D9), energy (D10), education (D11), security (D12), food and agriculture (D13), games / entertainment (D14), electronics (D15), and finally, chemistry (D16). From Figure 2, it is feasible to calculate that six domains cover $72.9 \%$ of all applications while the remaining $27.1 \%$ is covered by 10 domains.

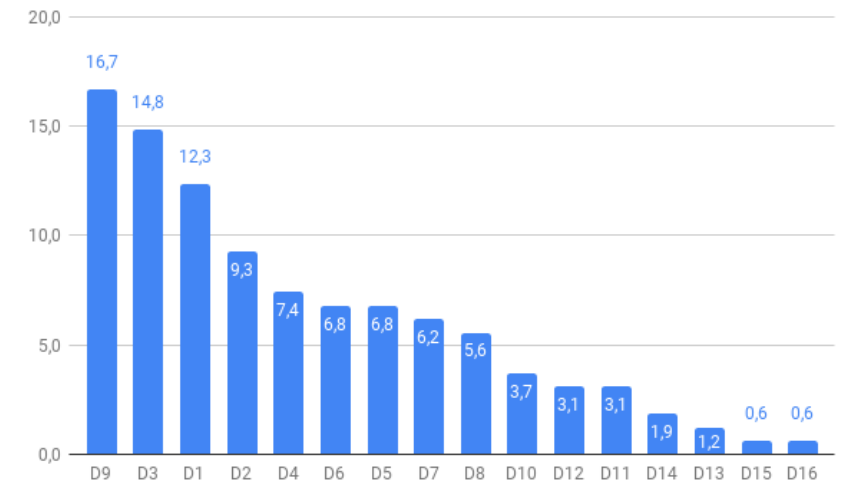

Figure 2: Column chart describing the values per domain as total, without separating ABMS from pure MAS implementations.

Finding 4.7. A) The top three domains on modelling and simulation were transport/traffic/ports (D2), healthcare/medicine/biology (D3), and logistics/manufacturing (D5); B) The top four domains for multi-agents implementations were health-care/medicine/biology (D3), information science (D1), logistics/manufacturing (D5), and business (D7).

\subsection{RQ6: What are the research gaps?}

By reading each of the included articles, it was possible to extract the gaps mentioned and found by the authors. As such, we were able to schematize the research gaps into two groups: the ones related to the paradigm itself paradigm itself (see Table 1), and those related to specific application domains or disciplines (see Table 2). Consequently, in Table 1 is possible to find gaps related to agent-oriented methodologies; coordination, cooperation and negotiation; modelling, developing, testing and debugging, and metrics; while Table 2 describes those related to specific domains (recycling, dynamic evacuation of pedestrians, hazard management, health-care, industry, logistics and manufacturing, machine learning, and Ambient Assisted living (AAL)) or specific areas within MAS (A-Teams, dynamic MAS and mobile agents, ABMS, evolutionary MAS, and self-organizing MAS).

Table 1: General Research Gaps

\begin{tabular}{|c|c|}
\hline Topics & Description of General Gaps \\
\hline $\begin{array}{l}\text { Agent- } \\
\text { Oriented } \\
\text { Methodologic }\end{array}$ & $\begin{array}{l}\text { - Absence of systematic methodology (Sturm, Dori, \& Shehory, 2010). } \\
\text { - None of the modeling languages is widely accepted and used (Sturm, Dori, \& Shehory, 2010). } \\
\text { - "It seems that existing agent-oriented methodologies focus on the development of new systems and not on other stages } \\
\text { and aspects within the system lifecycle. Even with reference to development process, not all phases are well supported" } \\
\text { (Sturm \& Shehory, 2014). } \\
\text { - "Another void is the lack of support for a paradigm shift to agent orientation. We believe that the latter is a grand } \\
\text { challenge for agent-oriented methodologies. That is, it is necessary-yet challenging-to justify the paradigm shift from } \\
\text { existing paradigms such as object-oriented, service-oriented, and business-process-oriented to the agent-oriented } \\
\text { paradigm" (Sturm \& Shehory, 2014). } \\
\text { - "While a number of modeling languages for constructing multi-agent systems (MASs) have been suggested, none of } \\
\text { them is widely accepted and used. A prominent reason for this is the gap between agent-oriented modeling languages and } \\
\text { the agent-based system modeling needs, including accessibility, flexibility, and expressiveness" (Sturm, Dori, \& Shehory, } \\
\text { 2010). } \\
\text { - "Put more effort into integrating agent-based methodologies and programming languages" (Dastani et al 2009). }\end{array}$ \\
\hline Coordination, & - "An open question is the analysis of coordination problems with larger number of agents" (Lang \& Fink, 2012). \\
\hline $\begin{array}{l}\text { Cooperation } \\
\text { and } \\
\text { Negotiation }\end{array}$ & $\begin{array}{l}\text { - "Some important problems in multi-agent system research are: how to enable the agents in the system to communicate } \\
\text { and interact with each other? what kind of communication language and protocol do they use? how to coordinate the } \\
\text { multiple agents in the system to achieve a common system goal through their communication and collaboration?" (Wu \& } \\
\text { Sun, 2010) }\end{array}$ \\
\hline & - "Study how to employ the debugging frameworks that are available for the embedded languages (such as Prolog or a \\
\hline
\end{tabular}




\begin{tabular}{|c|c|}
\hline Topics & Description of General Gaps \\
\hline Debugging & $\begin{array}{l}\text { Web Ontology Language }(\mathrm{OWL}) \text { )". (Dastani et al 2009). } \\
\text { - "A debugger for agent programming in general is needed" (Dastani et al 2009). } \\
\text { - "Recent developments in multi-agent programming languages have proposed specific programming constructs enabling } \\
\text { the implementation of social concepts such as norms, roles, obligations, and sanctions. Debugging such multi-agent } \\
\text { programs requires therefore specific debugging constructs to specify properties related to the social aspects and facilitate } \\
\text { finding and resolving defects involved in such programs" (Dastani et al 2015) } \\
\text { - "A debugger for multi-agent programs that run simultaneously on different platforms is also needed" (Dastani et al } \\
\text { 2015). } \\
\text { - "There are more challenges in debugging cognitive agents that need to be addressed. One of these is the fact that agents } \\
\text { are (usually) connected to an environment. Two problems need to be dealt with: (i) it can no longer be assumed that an } \\
\text { environment is deterministic which makes it difficult to reproduce a defect, and (ii) such environments typically cannot be } \\
\text { suspended instantly which makes it difficult to understand the context of a defect. Another problem is the fact that } \\
\text { debugging multiple agents at once is significantly more complicated than debugging a single agent. Finally, to further } \\
\text { analyse the effectiveness of a source-level debugger in agent programming in general, a detailed design of a source-level } \\
\text { debugger for other agent programming languages can be of interest. It would be specifically interesting to investigate the } \\
\text { effect of a more complicated decision cycle on the usability of a source-level debugger" (Koeman \& Hindriks, 2015) } \\
\text { - "Studies and work on testing should increase" (Leitão \& Vrba, 2011). } \\
\text { - "We believe that comprehensive automated unit testing is a critical first step in thorough testing of agent systems. } \\
\text { However, it is limited in what it tests for" (Zhang et al, 2011) } \\
\text { - "Further research in the area should focus on developing and testing more advanced coordination methods, which could } \\
\text { be used in real-time applications. Tests on large groups of real robots should also be performed" (Turek, 2010) } \\
\text { - "It is time to start paying more attention to the kind of support that a MAS developer needs to facilitate him or her when }\end{array}$ \\
\hline Developing & $\begin{array}{l}\text { engineering future MAS applications. It is important to identify the needs of a developer and make sure that a developer is } \\
\text { provided with the right tools for engineering MAS. For the same reason we should focus more on issues related to ease of } \\
\text { use, scalability and performance, and testing" (Hindriks, 2014) }\end{array}$ \\
\hline Modelling & $\begin{array}{l}\text { - "There is a need to bridge the gap between modeling and implementation, i.e., creating guidelines or rules for synthesis } \\
\text { of runnable agents from the abstract modeling components" (Lian et al, 2009) }\end{array}$ \\
\hline Metrics & - "The need for software metrics is now fully recognized" (Leitão \& Vrba, 2011). \\
\hline
\end{tabular}

Table 2: Domain-Specific Research Gaps

\begin{tabular}{|c|c|}
\hline Topics & Description of Domain-Specific Gaps \\
\hline Recycling & $\begin{array}{l}\text { - "Automate communication and cooperation of parties involved in the collection process" (Bezirgiannis \& Skaellariou, } \\
\text { 2011). } \\
\text { - "Lack of modern tools for managing the collection of recyclable resources" (Bezirgiannis \& Skaellariou, 2011). }\end{array}$ \\
\hline $\begin{array}{l}\text { Dynamic } \\
\text { evacuation of } \\
\text { pedestrians }\end{array}$ & $\begin{array}{l}\text { - "Improve the studies on: the impact of mixtures of groups and mixtures of spatial areas, stairway and exit capacities for } \\
\text { individuals with disabilities, the speed and mobility over different surfaces to increase the realism of the evacuation } \\
\text { models" (Manley, et al 2016). }\end{array}$ \\
\hline $\begin{array}{c}\text { Hazard } \\
\text { management }\end{array}$ & $\begin{array}{l}\text { - "Future studies should pay attention to encoding learning algorithms and reconstructing the hazard-management } \\
\text { processes in historical events (e.g. Hurricane Katrina). Constructing geographic ontologies is so challenging that no } \\
\text { commonly shared ontology is yet available. Managing large-scale natural hazard events often involves multiple decision } \\
\text { makers in different disciplines or cultures across regions or countries; thus building a sharable ontology is crucial for } \\
\text { sharing information and reducing misunderstanding or miscommunication" (Yu et al, 2009). }\end{array}$ \\
\hline Health-care & $\begin{array}{l}\text { - "The majority of work done is domain specific like patient scheduling" (Benhajji, N., et al 2015). } \\
\text { - "Consider the advancements in biomedical sensor nodes capable" (Shakshuki \& Reid, 2015). } \\
\text { - "Increase the amount of multi-agent applications design to prolong the independent lifestyle of seniors" (Shakshuki \& } \\
\text { Reid, 2015). }\end{array}$ \\
\hline & $\begin{array}{l}\text { - "Within the steel industry, a steel automation system is needed to represent distribution and integration. Using multi- } \\
\text { agent systems depicts such capabilities." (Zarandi, et al. 2009). }\end{array}$ \\
\hline & $\begin{array}{l}\text { - "It would be useful to provide demonstrators running in industry and return of investment (ROI) analysis, not only in } \\
\text { terms of development costs but also in terms of operation and maintenance costs" (Leitão \& Vrba, 2011) } \\
\text { - "Lack of widely available industrial-strength multi-agent system toolkits" (Jennings et al, 1998) } \\
\text { - "the main future trends for industrial agents are centered on: } \\
\text { - Consideration of mature tools and development engineering methodologies that simplifies the engineer of agent-based } \\
\text { systems (integrating the design, verification, simulation, and deployment phases) and also supporting technologies to run } \\
\text { agent-based solutions, e.g., at low level control. }\end{array}$ \\
\hline Ind & $\begin{array}{l}\text { - Consider the integration with other technologies, namely the integration with the low-level control running in state-of- } \\
\text { the-art PLCs running IEC61131-3 control programs or using the IEC } 61499 \text { standard since the direct connection with } \\
\text { physical devices is usually mandatory in industrial environments. Aiming to address other integration vectors, namely the } \\
\text { IT-vertical integration, the combination with web services should be considered to address the questions related to the } \\
\text { interoperability. }\end{array}$ \\
\hline & $\begin{array}{l}\text { - Consideration of bio-inspired techniques, and particularly self-organization and emergent behaviour, enhancing multi- } \\
\text { agent systems to support the engineer of more robust, adaptive, reconfigurable and responsive systems. }\end{array}$ \\
\hline & $\begin{array}{l}\text { - As highlight by industrial experts, the consideration of standardization issues in the development of agent-based } \\
\text { solutions, namely the IEC } 61131-3 \text { and ISA } 95 \text { standards, is crucial. Note that the Foundation for Intelligent Physical } \\
\text { Agents (FIPA) is a standard for the development of multi-agent systems, but it misses many of the particularities imposed } \\
\text { by industrial environments" (Leitão \& Vrba, 2011) }\end{array}$ \\
\hline Logi & $\begin{array}{l}\text { - "FIPA misses many of the particularities imposed by industrial environments" (Leitão \& Vrba, 2011). } \\
\text { - "New architectures of manufacturing automation systems are required to implement a smart factory" (Gohner } \\
\text { \&Weyrich, 2014). }\end{array}$ \\
\hline
\end{tabular}




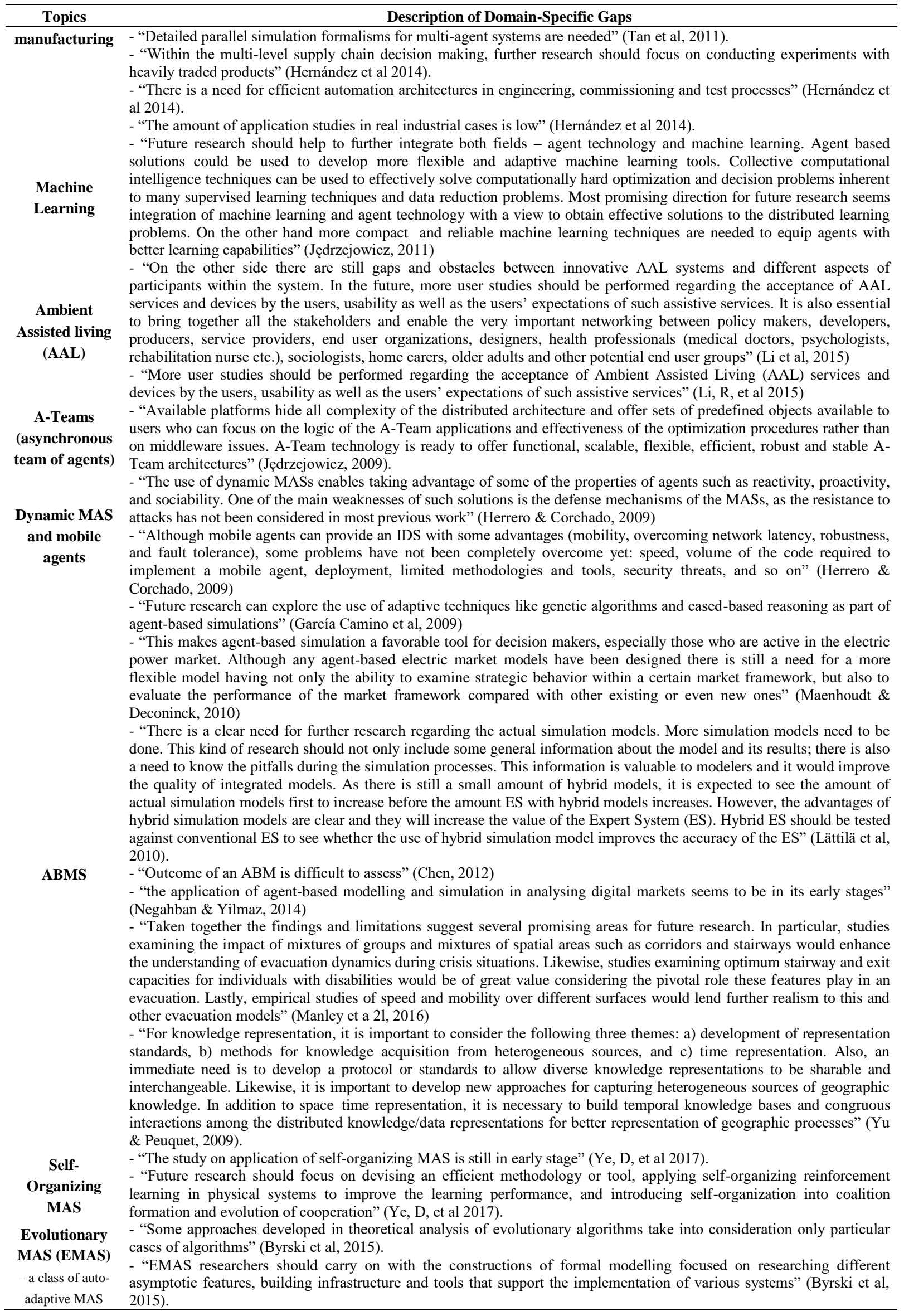


Finding 4.8. A) There is a lack of unified tools (methodologies, framework, among others) that developers can use to implement a MAS; B) The work done is mostly domain-specific; C) the MAS community should build ties with the industry; D) a detailed design of a source-level debugger for other agent programming languages can be of interest; E) There is a need to bridge the gap between modelling and implementation; F) FIPA misses many of the particularities imposed by industrial environments; G) Constructing geographic ontologies is so challenging that no commonly shared ontology is yet available.

Following finding 4.8, it is feasible to say that the MAS community should keep working on closing the gaps shown in Tables 1 and 2. Consequently, these Tables not only demonstrate that there has been advances in specific fields, but the resolution of the most critical or unresolved aspects of the domains, whose resolution would help to mature the paradigm, remain pending.

\section{Threats to validity}

A few articles from 2017 were not available to download while we were performing the analysis of each paper, from which some papers could be added to that period modifying a little bit the amount of papers published that year, and that is the main reason because we did not include them in Fig. 1. Likewise, it is feasible to point out that the trend shown in Fig. 1 might be influenced on the loose of interest in that particular publication (S3), but it is compensated by the validity of the rest of the articles of the start set (2014 and 2015). Consequently, in this article, the factors, which jeopardize internal validity, are as follows: a change within the followed method may produce changes in the outcomes, a broader range of years, and the inclusion of the papers that were not available when the SLR was carried out. Also, in order to provide a fearful basis onto the automated search, one of the authors performed a search on online databases to obtain the amount of articles for 2015 (based on Fig. 1) with "survey multi-agent systems" on publication title. As a result, we obtained two articles from ACM and none from Springer.

One of the articles was excluded based on the title, and the second one was a tutorial on NetLogo (excluded as well). Consequently, GS were a valid source to conduct the search. Nevertheless, one of the main limitations of this study is that maybe some good papers were excluded because they were not referenced in any of the four papers that made up the start set or that did not cite at least one of those four papers. For that, another iteration might be sufficient to fully complete the start set. Or even, including text-mining techniques to improve the search. For these reasons it is not possible to generalize the results, but it is necessary to understand that they respond to a partial sample, but not negligible.

\section{Discussion}

Different authors [3, 8] mentioned the need of justifying the necessity for a 'paradigm-shift' from existing paradigms to the agent-oriented paradigm; which it will require a change within the basic concepts and practices within each discipline and domain of application. In this context, a shift will be supported if and only if there is a broad adoption and acceptance of the technology itself. So, is the MAS community using solely AOSE components? Or is it using a combination of the object- and the agent-oriented paradigms? Is the latter ready to take-off individually? There is no doubt about the need for a new paradigm evidenced in the number of domains in which MAS have been applied, but does it satisfies all the needs to face highly complex applications?

Back in 1998, there were two major technical impediments to the widespread adoption of agent technology [30], where the first one concerned to the absence of a systematic and unified methodology, while the second one was related to the lack of industrial-strength MAS toolkits. Up today, there is a diversity of methodologies that many of them share a common basis, and new methodologies for specific domains have been developed. However, none of the modelling languages is widely accepted and used [31]. The actual agent-oriented methodologies are mainly focused on the development of systems, but without contemplating other phases or stages [8]. Consequently, further progress must be made so that MAS technologies can cover the entire software life cycle [32].

In addition, the life cycle models should support reusability. The reuse of code and concepts are important when developing MAS, and Schumann [33] presented an approach to effective and efficiently select mechanisms to coordinate plans among agents. The following question arises at this point: can we expect from MAS a paradigm that covers all phases of the life cycle of the systems or is it just an extension of OO, with complexity, autonomy, parallelism, feedback from the environment, and emerging behaviour; applicable in certain parts of a system or phases of the life cycle?

Several studies explained that Java and JADE are the most used programming language and framework respectively, within the agent-oriented applications [9], but both of them comes from the OOP. Even though JACK is the most used agent-oriented framework, JADE is applied in a higher amount of applications. Based on our knowledge, we recommend the programmers to perform a proper analysis before implementing JADE, due to the fact that is deprecated. Likewise, programmers who are up to new challenges (considering the learning curve) might select directly a multi-agent programming modelling environment like NetLogo. However, is it fully justified the 
learning curve? From previous experience of the authors, it is feasible to say that the generated code is difficult to maintain when it evolves and is useful for simple simulations.

The second major impediment was related to the lack of widely available MAS toolkits powerful enough to be used in industrial settings [30]. There are several challenges that have a huge impact on the acceptance of industrial agents [34,35]. As such, a shift is needed within industrial environments for automation vision because it portrays a decay of the applicability, and real deployment of the agent technology [35]. Even though the industrial adoption and application of agent technology is endorsed by different initiatives by International Technical Committees and $\mathrm{R} \& \mathrm{D}$ projects, the contemplation of standardization matters within the development of agent-based solutions is decisive. In the MAS context, the integration and feedback from the industries and business that have already used or develop a MAS, would be extremely useful to improve the existing bonds and create new ones on industrial settings. As Muller and Fischer mentioned (...) "the strong presence of Spain and Italy for highly mature applications is based on the strong industrial players Telefonica $T+D$ and Telecom Italia” (...) [9].

Nowadays, and as a part of the regular progress of the Software Engineering field, the requirement for software metrics is now fully recognized by the community [36]. Even though the reasons for defining and implementing metrics are independent of the chosen paradigm, the latter have an impact on the set of metrics to employ [37]. Also, within the advances in technologies, the application of agent-based solutions to machine learning have proven to harvest a synergetic effect within the power of achieving cooperative solutions implemented by interactions between agents. For that, (...) "machine learning can be seen as a prime supplier of learning capabilities for agent and multi-agent systems, while agent technology has brought to machine learning several capabilities including parallel computation, scalability and interoperability" (...) [38].

Finally, as seen in Tables 1 and 2, it is possible to say that although the paradigm tended lines of growth with respect to its origins, today there are still gaps as the absence of a systematic methodology and toolkits for the industry; which are drawback points at the moment a project leader decides whether to implement a multi-agent system or not.

\section{Conclusions}

In the present article, a systematic literature review was carried out around six research questions, following a wellknown guideline [12], and complemented with the snowballing approach [13], in order to understand the progresses of Multi-Agent Systems, from 2009 to the present days. After applying the snowballing approach of the 3522 candidates, only 279 remained, which were estudied in the industrial, and academic context.

We have obtained 67 representative authors from 49 countries, who have obtained a range between 41 and 248 citations per each related to the amount of articles within the 279 articles analyzed, and who have published in 102 journals, 111 conferences, 11 symposiums and 4 congresses. Almost $30 \%$ of the applications achieved one of the three levels of maturity presented in [9], mostly in academia but within the cooperation with industry and public administration. These numbers make up the first topic that makes a consistent and repeating feature in the answers to all the research questions: dispersion. Likewise, we have found a high number of domains in which MAS have been applied which lead us to understand that there is not a single possible domain of application, which gives us variety and dynamism. This dispersion also means that the field is growing, is becoming more specific and bounded, but how do we manage to unify it? How do we support the learning curve of developers?

Besides, what are the causes of the decrease in the general number of publications? It corresponds to a depletion of the paradigm, to the lack of adequate tools, a low level of industrial assimilation, to the non-integration of the different branches that emerged from the growth and specification of the field? Or is growing domain by domain? MAS is an area that, although it requires work to close and unify the threads, makes it possible to tackle complex systems in a changing and increasingly complex world.

The research gaps show that there is a need to fully close existing and old topics within the MAS field: ties with the industry (as well as a widely available MAS toolkit) and a systematic methodology of developing. As such, if the community wants to fully integrate multi-agent systems within industrial settings, they should develop industrial toolkits that adapt to the particularities in force in the industry, and increase not only the amount of applications but the applicability in real life. Likewise, the MAS community should learn from the history of the object-oriented paradigm. As such, if there is no industry acceptation then there is no growth and sustainable maturity to be a true paradigm.

Even though the improvement in computing power (...) "has increased the scope of MAS employability in realworld applications" (...) [39], as Dawson-Díaz and Vega-Zepeda [40] stand, and following Tables 1 and 2, we can conclude that there is no standardized process for creating Multi-Agent Systems, and the fact that many methodologies do not cover the whole lifecycle, might lead to a non-allowance of using this technology within the industry. Is the author's beliefs that a standardization of terminology and methodology [8] would lead to the maturity of the paradigm, as years ago UML and the Unified Process (UP) had an impact on the object-oriented 
paradigm; recalling that at the time they participated in the unification of language and methodology people from academia and industry.

\section{Acknowledgements}

This work was supported by a research grant from Universidad Austral.

\section{References}

[1] N. Jennings, K. Sycara and M. Wooldridge, "A roadmap of agent research and development", Autonomous Agents and Multi-Agent Systems, vol. 1, no. 1, pp. 7-38, 1998.

[2] M. Wooldridge, An introduction to multiagent systems, 2nd ed. John Wiley \& Sons, 2009.

[3] K. Hindriks, "The shaping of the agent-oriented mindset." International Workshop on Engineering MultiAgent Systems, pp. 1-14, 2014.

[4] Ferber, J., \& Weiss, G. (1999). Multi-agent systems: an introduction to distributed artificial intelligence (Vol. 1). Reading: Addison-Wesley.

[5] Q. Bai and N. Fukuta, Advances in Practical Multi-Agent Systems, Berlin, Heidelberg: Springer-Verlag, 2011.

[6] M. Aulinas, C. Turon and M. Sànchez-Marrè, "Agents as a decision support tool in environmental processes: the state of the art", Advanced Agent-Based Environmental Management Systems, U. Cortés and M. Poch, Ed. Birkhäuser Basel, pp. 5-35, 2018.

[7] O. Shehory and A. Sturm, "A brief introduction to agents", Agent-Oriented Software Engineering, Springer Berlin Heidelberg, pp. 3-11, 2014.

[8] A. Sturm and O. Shehory, "The landscape of agent-oriented methodologies", Agent-Oriented Software Engineering, Springer Berlin Heidelberg, pp. 137-154, 2014.

[9] J. P. Müller and K. Fischer, “Application Impact of Multi-Agent Systems and Technologies: A Survey”, AgentOriented Software Engineering: Reflections on Architectures, Methodologies, Languages, and Frameworks. Springer, pp. 27-53, 2014.

[10] D. Isern and A. Moreno, "A systematic literature review of agents applied in healthcare", Journal of medical systems, 40(2), 43, 2016.

[11] Ge, X., Han, Q. L., Ding, D., Zhang, X. M., \& Ning, B. (2018). A survey on recent advances in distributed sampled-data cooperative control of multi-agent systems. Neurocomputing, 275, 1684-1701.

[12] B. Kitchenham and S. Charters, Guidelines for Performing Systematic Literature Reviews in Software Engineering, version 2.3, Technical Report, 2007.

[13] C. Wohlin, "Guidelines for snowballing in systematic literature studies and a replication in software engineering", 18th International Conference on Evaluation and Assessment in Software Engineering, ACM, p. $38,2014$.

[14] M. Falco and G. Robiolo, "A Systematic Literature Review in Multi-Agent Systems: Patterns and Trends", XLV Conferencia Latinoamericana de Informática, Centro Latinoamericano de Estudios de Informática (CLEI), $\quad$ Panamá. $\quad 30 \quad$ Septiembre $\quad-\quad 4 \quad$ Octubre $\quad 2019$, IEEE. http://clei2019.utp.ac.pa/storage/app/uploads/public/5d8/cf6/967/5d8cf69670cdc021709763.pdf

[15] A. Carrera and C. A. Iglesias, "A systematic review of argumentation techniques for multi-agent systems research”, Artificial Intelligence Review, 44(4), pp. 509-535, 2015.

[16] S. A. Mostafa, M. S. Ahmad and A. Mustapha, "Adjustable autonomy: a systematic literature review", Artificial Intelligence Review, pp. 1-38, 2017.

[17] J. L. Morales, P. Sánchez and D. Alonso, "A systematic literature review of the teleo-reactive paradigm", Artificial Intelligence Review, 42(4), pp. 945-964, 2014.

[18] D. Blanes, E. Insfran and S. Abrahão, "Requirements engineering in the development of multi-agent systems: a systematic review", International Conference on Intelligent Data Engineering and Automated Learning, Springer Berlin Heidelberg, pp. 510-517, 2009. 
[19] J. Juziuk, D. Weyns and T. Holvoet, “Design patterns for multi-agent systems: a systematic literature Review”, Agent-Oriented Software Engineering, Springer Berlin Heidelberg, pp. 79-99, 2014.

[20] A. Negahban and L. Yilmaz, "Agent-based simulation applications in marketing research: an integrated review”, Journal of Simulation, 8(2), pp. 129-142, 2014.

[21] M. Wooldridge and N. R. Jennings, N.R., "Agent theories, architectures, and languages: A survey", in M. J. Wooldridge and N.R. Jennings (eds) Intelligent Agents. ATAL 1994. Lecture Notes in Computer Science (Lecture Notes in Artificial Intelligence), vol. 890, Springer, Berlin, Heidelberg, 1995.

[22] J. Cohen, "Weighted Kappa: Nominal Scale Agreement Provision for Scaled Disagreement or Partial Credit," Psychological Bulletin, vol. 70, no. 4, p. 213, 1968.

[23] D. Giustini and M. N. K. Boulos, "Google Scholar is not Enough to be Used Alone for Systematic Reviews," Online journal of public health informatics, vol. 5, no. 2, p. 214, 2013.

[24] T. Balke, B. Hirsch and M. Lützenberger, "Assessing agent applications-r\&D vs. R\&d”, Multiagent systems and applications, Springer Berlin Heidelberg, pp. 1-20, 2013.

[25] Y. Abushark, T. Miller, J. Thangarajah, M. Winikoff and J. Harland, "Requirements specification via activity diagrams for agent-based systems", Autonomous Agents and Multi-Agent Systems, 31(3), pp. 423-468, 2017.

[26] V. Gorodetsky, O. Karsaev, V. Samoylov and V. Konushy, "Support for analysis, design, and implementation stages with MASDK", International Workshop on Agent-Oriented Software Engineering, Springer, Berlin, Heidelberg, pp. 272-287, 2008.

[27] Y. Hedin and E. Moradian, "Security in Multi-Agent Systems", Procedia Computer Science, 60, pp. 1604$1612,2015$.

[28] M. Dastani, "A survey of multi-agent programming languages and frameworks", Agent-Oriented Software Engineering, Springer Berlin Heidelberg, pp. 213-233, 2014.

[29] M. Falco, L. Bauret and G. Robiolo, "HeartCare: an Agent Oriented Architecture Implemented with Actors", XXV Congreso Argentino de Ciencias de la Computación (CACIC 2019). Universidad Nacional de Río Cuarto, 14 al 18 de Octubre de 2019. Río Cuarto, Córdoba, Argentina.

[30] N. R. Jennings, K. Sycara and M. J. Wooldridge, "A Roadmap of Agent Research and Development", Autonomous Agents and Multi-Agent Systems 1, 1, ACM, pp. 7-38, 1998.

[31] A. Sturm, D. Dori and O. Shehory, "An object-process-based modeling language for multiagent systems", IEEE Transactions on Systems, Man, and Cybernetics, Part C (Applications and Reviews), 40(2), pp. 227-241, 2010.

[32] J. Zhang, L. Cheng and H. Wang, “A multi-agent-based decision support system for bankruptcy contagion effects”, Expert Systems with Applications, 39(5), pp. 5920-5934, 2012.

[33] R. Schumann, “Engineering Coordination”, KI-Künstliche Intelligenz, 26(1), Springer, pp. 87-90, 2012.

[34] P. Leitão and P. Vrba, "Recent developments and future trends of industrial agents", Holonic and Multi-Agent Systems for Manufacturing, Springer, pp. 15-28, 2011.

[35] S. Karnouskos and P. Leitão, "Key Contributing Factors to the Acceptance of Agents in Industrial Environments”, IEEE Transactions on Industrial Informatics, 13(2), pp. 696-703, 2017.

[36] Guidelines for the Application of ISO 9001 to the Development, Supply and Maintenance of Software, ISO/IEC, June 1991.

[37] F. B. e Abreu and R. Carapuça, Candidate metrics for object-oriented software within a taxonomy framework. Journal of Systems and Software, 26(1), pp. 87-96, 1994.

[38] I. Czarnowski and P. Jędrzejowicz, "Machine Learning and Multiagent Systems as Interrelated Technologies", Agent-Based Optimization, Springer Berlin Heidelberg, pp. 1-28, 2013.

[39] P. G. Balaji and D. Srinivasan, “An introduction to multi-agent systems”, Innovations in multi-agent systems and applications-1, Springer Berlin Heidelberg, pp.1-27, 2010.

[40] N. Dawson-Díaz and V. Vega-Zepeda, Lifecycle coverage analysis via multi-agent system methodology. In 6th International Conference on Software Process Improvement (CIMPS), pp. 1-7, IEEE, 2017, October. 
Appendix (if necessary) 